\title{
BICO MPPT: A Faster Maximum Power Point Tracker and Its Application for Photovoltaic Panels
}

\author{
Hadi Malek ${ }^{1}$ and YangQuan Chen ${ }^{2}$ \\ ${ }^{1}$ Electrical \& Computer Engineering Department, Utah State University, Logan, UT 84321, USA \\ ${ }^{2}$ MESA Lab, School of Engineering, University of CA, Merced, California 95343, USA \\ Correspondence should be addressed to Hadi Malek; hadi.malek@aggiemail.usu.edu
}

Received 29 May 2013; Accepted 3 January 2014; Published 27 February 2014

Academic Editor: Ismail H. Altas

Copyright (c) $2014 \mathrm{H}$. Malek and Y. Chen. This is an open access article distributed under the Creative Commons Attribution License, which permits unrestricted use, distribution, and reproduction in any medium, provided the original work is properly cited.

\begin{abstract}
This paper develops a maximum power point tracking (MPPT) algorithm to optimize photovoltaic (PV) array performance and make it more compatible to rapidly varying weather conditions. In particular, a novel extremum seeking controller (ESC), which uses a Bode ideal cutoff (BICO) filter in its structure, is designed and tested on a simulated PV array. The new algorithm is compared against the commonly used ESC MPPT algorithm with first-order filters. The BICO extremum seeking controller achieves transient rise to the MPP faster than the common extremum seeking MPPT, which is the faster and more robust method among all other methods. This claim has been discussed and proved mathematically in this paper, in addition to simulation illustrations. This faster extremum seeking algorithm enables PV systems to detect rapid variations in the environmental conditions like irradiation and temperature changes.
\end{abstract}

\section{Introduction}

The PV cells exhibit a nonlinear $V-I$ characteristics as shown in Figure 1 and their power output mainly depends on the nature of the connected load. Since connecting the load directly to the PV system results in a poor overall efficiency, to minimize the life cycle cost of any PV system, increasing the efficiency by changing the operating point of the system using an intermediate maximum power point tracker (MPPT) is desirable.

MPPT controls the output current and voltage and consequently output power of the PV panel adaptively to maintain maximum efficiency and better performance in the presence of environmental variations. Typically MPPT algorithms are implemented on a solar array using a switching power converter; for instance, in a grid-tied inverter, the solar array charges a capacitor and then the current is switched out of the capacitor at an optimal varying duty cycle in order to extract maximum power from the PV array.

A number of solar power converter architectures with MPPT are discussed in the literature $[1,2]$. As discussed in these works, convergence speed is one of the most important features among all different MPPT algorithms. Brunton et al. have pointed out in their paper that, "as irradiance decreases rapidly, the $I V$ curve shrinks and the MPV and MPI decrease. If the MPPT algorithm does not track fast enough, the control current or voltage will fall off the $I V$ curve" [3]. Consequently, any improvement in the rise time of MPPT improves the reliability of the system and increases the power extraction and efficiency of the whole system.

1.1. Maximum Power Point Tracking Algorithms. There are many different maximum power point tracking techniques for photovoltaic systems which are well established in the literature [4-11]. These techniques vary in many aspects as simplicity, convergence speed, digital or analogical implementation, sensors required, cost, range of effectiveness, and other aspects. In analog world, short current (SC), open voltage $(\mathrm{OV})$, and $\mathrm{CV}$ are good options for MPPT; otherwise with digital circuits that require the use of microcontroller, Perturb and Observe (P\&O), Incremental Conductance (IC), and temperature methods are easy to implement [12]. Table 1 and Figure 2 present the comparison among different MPPT 


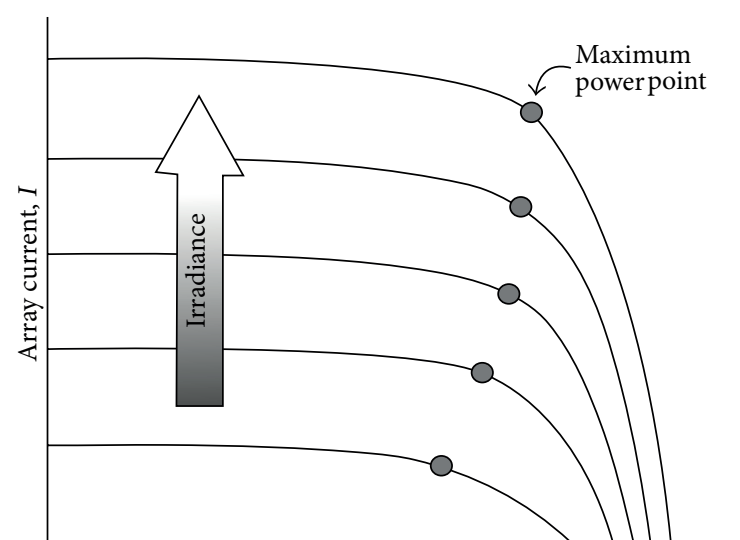

Terminal voltage, $V$

FIGURE 1: $I-V$ curves at various solar irradiances [3].

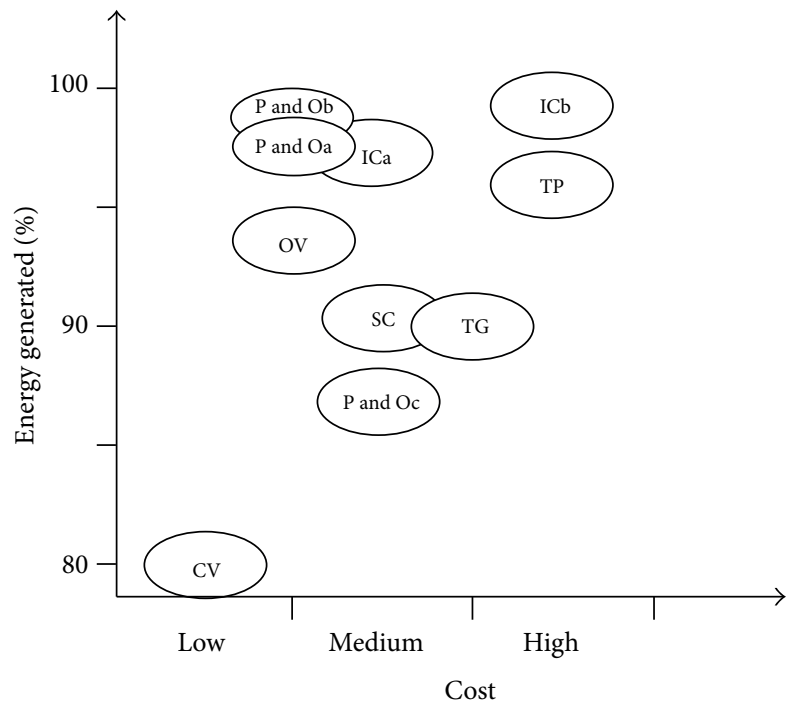

FIGURE 2: Comparison of the MPPT methods [12].

methods considering the costs of sensors, microcontroller, and additional power components and also their efficiencies. In this table, $\mathrm{A}$ means absence, $\mathrm{L}$ means low, $\mathrm{M}$ means medium, and $\mathrm{H}$ means high.

Currently, the most popular and workhorse MPPT algorithm is $\mathrm{P} \& \mathrm{O}$, because of its balance between performance and simplicity. However, it suffers from the lack of speed and adaptability which is necessary for tracking the fast transients under varying environmental conditions [3]. Recently, another adaptive algorithm, called extremum seeking control, has been developed [13] to overcome these weaknesses.

1.2. Extremum Seeking Control. A promising new robust MPPT algorithm is the method of extremum seeking (ES) control, which carries all $\mathrm{P} \& \mathrm{O}$ 's benefits like simplicity and performance, and in addition, improves its weaknesses [3]. Figures 3 and 4 present the comparison between ESC and $\mathrm{P} \& \mathrm{O}$, where the inverter controls the current and voltage

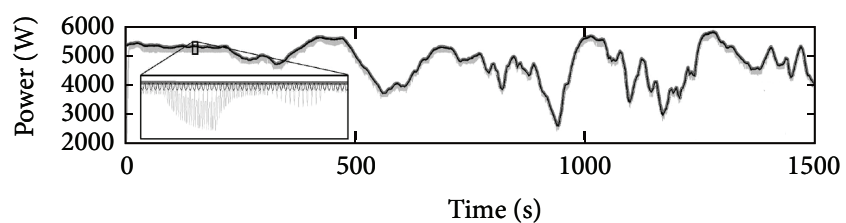

(a)

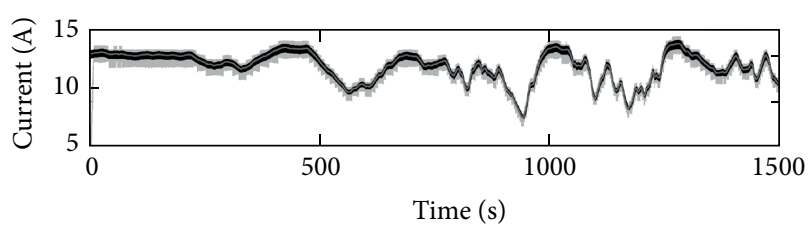

(b)

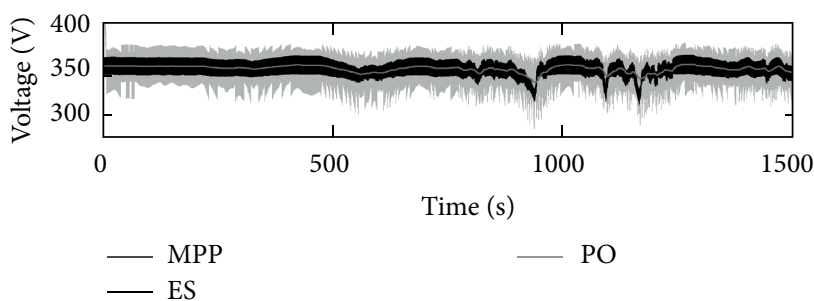

(c)

Figure 3: Comparison of current controlled P\&O and ESC MPPT controller [3].

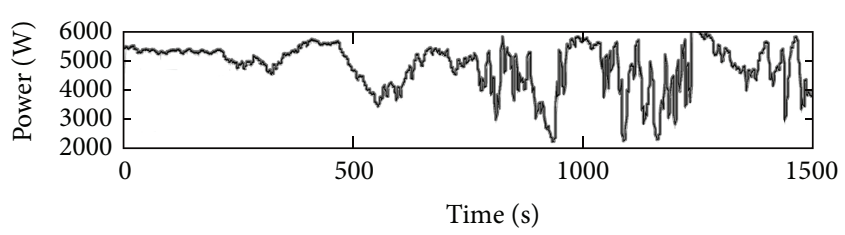

(a)

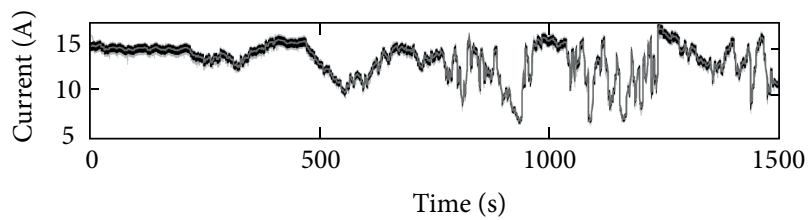

(b)

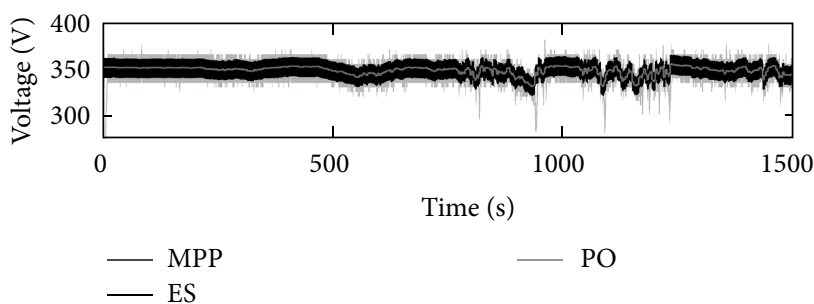

(c)

FIgURE 4: Comparison of voltage controlled P\&O and ESC MPPT controller [3]. 
TABLE 1: MPPT methods comparison [12].

\begin{tabular}{lcccc}
\hline MPPT algorithm & Additional components & Sensors & Micro-Controller & Total \\
\hline CV & $\mathrm{A}$ & $\mathrm{L}$ & $\mathrm{A} / \mathrm{L}$ & $\mathrm{L}$ \\
$\mathrm{SC}$ & $\mathrm{H}$ & $\mathrm{M} / \mathrm{L}$ & $\mathrm{M}$ \\
OV & $\mathrm{H}$ & $\mathrm{L} / \mathrm{M}$ & $\mathrm{A} / \mathrm{L}$ & $\mathrm{L} / \mathrm{M}$ \\
P and Oa & $\mathrm{A}$ & $\mathrm{M}$ & $\mathrm{L}$ & $\mathrm{L} / \mathrm{M}$ \\
P and Ob & $\mathrm{A}$ & $\mathrm{M}$ & $\mathrm{M}$ & $\mathrm{L} / \mathrm{M}$ \\
P and Oc & $\mathrm{A}$ & $\mathrm{M}$ & $\mathrm{M}$ & $\mathrm{M}$ \\
IC & $\mathrm{A}$ & $\mathrm{M}$ & $\mathrm{M}$ & $\mathrm{M}$ \\
TG & $\mathrm{A}$ & $\mathrm{M} / \mathrm{H}$ & $\mathrm{M} / \mathrm{H}$ & $\mathrm{M}$ \\
TP & $\mathrm{A}$ & $\mathrm{H}$ & $\mathrm{H}$ \\
\hline
\end{tabular}

according to the set-points which are provided by these two MPPT algorithms.

The power, current, and voltage are plotted versus time for the ESC and $\mathrm{P} \& \mathrm{O}$ algorithms as well as the true maximum power of the system.

P\&O and ESC methods oscillate closely around the real maximum power voltage, as seen in the power versus time plot. Obviously, the ESC method rises to the MPP orders of magnitude more rapidly than the $\mathrm{P} \& \mathrm{O}$.

ESC MPPT has some advantages from hardware implementation point of view. Brunton et al. have mentioned in their paper that "the ripple-based ES algorithm has good MPPT performance over a range of inverter capacitor sizes. Typically, the choice of capacitor is expensive because it must be well characterized and large enough to maintain a small ripple. However, because the ES control signal exploits the natural inverter ripple, a smaller capacitor allows the tracking of rapid irradiance changes."

ESC for peak power point tracking method has been successfully applied to biochemical reactors [14, 15], ABS control in automotive brakes [16], variable cam timing engine operation [17], electromechanical valves [18], axial compressors [19], mobile robots [20], mobile sensor networks [21, 22], optical fibre amplifiers [23], and so on. A good survey of the literature on this topic prior to 1980 can be found in [24] and a more recent overview can be found in [25]. Astrom and Wittenmark rated extremum seeking as one of the most promising adaptive control methods [26].

Since extremum seeking control has better features and performance compare to $\mathrm{P} \& \mathrm{O}$ which is the best known MPPT algorithm, in this paper, the improvement of better than the best MPPT algorithm has been investigated.

\section{Basic Regular Extremum Seeking Algorithm}

As shown in Figure 5, ESC algorithm employs a slow periodic perturbation, $\sin (\omega t)$, which is added to the estimated signal, $\widehat{\theta}$. If the perturbation is slow enough, the plant appears as a static map $y=f(\theta)$ and its dynamics do not interfere with the peak seeking scheme [13]. If $\hat{\theta}$ is on either side of $\theta^{*}$, which is the optimal point, the perturbation signal $a \sin (\omega t)$, will create a periodic response of $y$ which is either in phase or out of phase with $a \sin (\omega t)$. The high-pass filter eliminates the "DC component" of $y$. Thus $a \sin (\omega t)$ and high-pass filter will

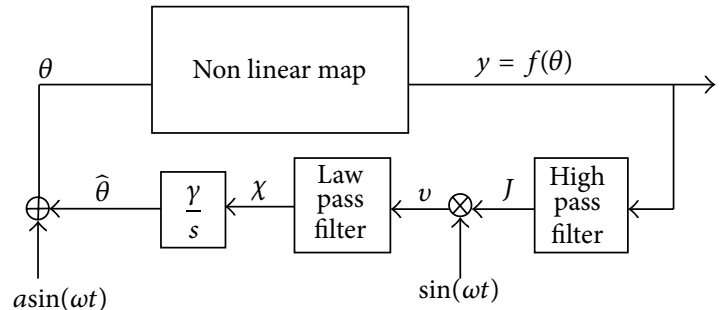

FIGURE 5: Extremum seeking algorithm scheme.

be approximately two sinusoids which are in phase if $\widehat{\theta}<\theta^{*}$ or out of phase if $\hat{\theta}>\theta^{*}$.

The integrator $\hat{\theta}=(\gamma / s) \chi$ approximates the gradient update law $\dot{\hat{\theta}}=k\left(a^{2} / 2\right)(f)^{\prime}(\widehat{\theta})$ which tunes $\hat{\theta}$ to $\theta^{*}[13]$. System of Figure 5 can be summarized in mathematical equations as follows:

$$
\begin{gathered}
y=f(\widehat{\theta}+a \sin (\omega t)), \\
\dot{\hat{\theta}}=-\gamma \chi, \\
\chi=v * \mathfrak{Q}^{-1}\left\{\mathrm{H}_{\mathrm{LPF}}(s)\right\}, \\
v=\left[y * \mathfrak{Q}^{-1}\left\{\mathrm{H}_{\mathrm{HPF}}(s)\right\}\right] \sin (\omega t),
\end{gathered}
$$

where $*$ is the convolution operator and $\mathfrak{Q}^{-1}$ is the inverse Laplace transform operator. The transfer functions for $\mathrm{H}_{\mathrm{HPF}}$ and $\mathrm{H}_{\mathrm{LPF}}$ in the regular SISO ESC scheme are $s /\left(s+\omega_{h}\right)$ and $\omega_{l} /\left(s+\omega_{l}\right)$ respectively, where $\omega_{l}<\omega<\omega_{h}$ [25]. This model will be used for stability analysis in this paper.

In the following sections, after introducing BICO filter $[27,28]$, the advantages of using this filter in the ESC algorithm from the stability and robustness point of view will be discussed.

\section{BICO Extremum Seeking Control MPPT}

In this section we are going to introduce a filter which was strongly favored by Bode [29], called Bode's ideal cutoff 


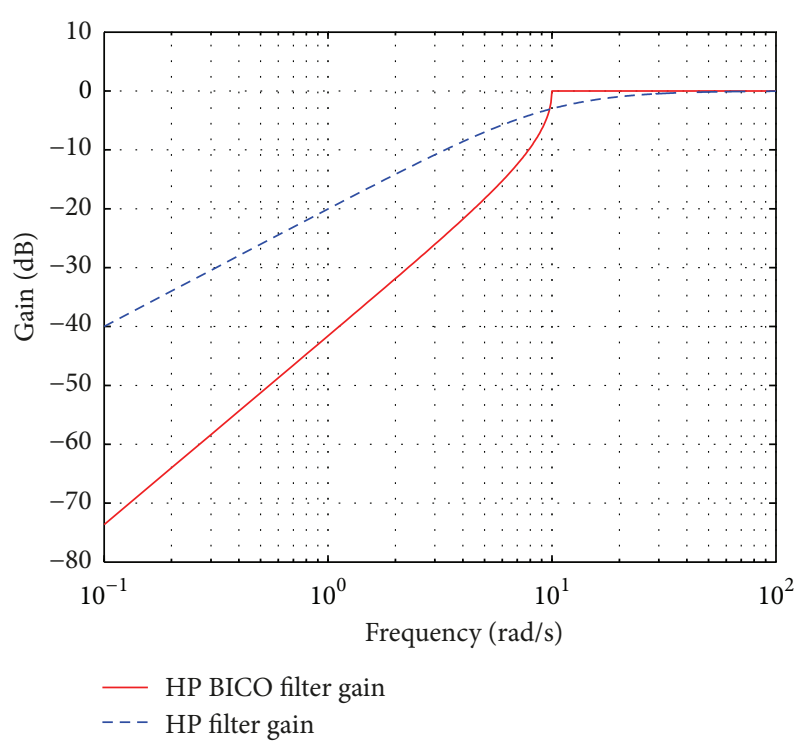

FIGURE 6: High-pass BICO and regular high-pass filters.

characteristic (BICO) filter $[27,28]$. The general transfer function of low-pass BICO filter is,

$$
G_{\mathrm{LP}-\mathrm{BICO}}(s)=\frac{k}{\left(s / \omega_{c}+\sqrt{1+\left(s / \omega_{c}\right)^{2}}\right)^{r}}, \quad r \in \mathbb{R}^{+} .
$$

Its corresponding time response in the special case $\left(\omega_{c}=\right.$ $1 \mathrm{rad} / \mathrm{s}$ ) was derived by Oberhettinger and Baddi [30], but the time response of (2) is presented as,

$$
g_{\mathrm{BICO}_{\mathrm{LP}}}(t)=k \frac{r J_{r}\left(\omega_{c} t\right)}{\omega_{c} t},
$$

where $J_{r}$ is the $r$ th order Bessel function.

By replacing $s$ by $1 / s$, high-pass BICO filter is obtained. Figure 6 compares the frequency response of high-pass BICO filter and regular high-pass filter $\left(s /\left(s+\omega_{c}\right)\right)$ with the same cutoff frequency of $\omega_{c}=10 \mathrm{rad} / \mathrm{s}$.

As seen in this figure, BICO filter has a sharp edge in its cutoff frequency. This great feature causes almost no attenuation for frequencies higher than $\omega_{c}$ and a large attenuation in the lower frequencies. Therefore the behavior of this filter is close to an "ideal" filter. This sharp edge presents in the low-pass BICO filter. By combining high-pass and low-pass BICO filters, the band-pass BICO filter with sharp edges in both sides can be obtained as well.

\section{Stability Analysis of Extremum Seeking Control}

4.1. Mathematical Modeling of ESC Scheme. The stability analysis of ESC algorithm has been investigated in [13, 31-34]. In these literatures traditional extremum seeking control with regular first-order filters has been considered. In this paper, we have considered the similar stability analysis approach as [34] to compare the stability and robustness of BICO-ESC and regular SISO ESC. The difference between this work and [34] is that in this paper, the stability analysis has been done by considering both low-pass and high-pass filters in the ESC structure but in [34] authors have investigated the stability of simplified ESC with only low-pass filter in its structure.

According to the previous discussion, the nonlinear map in the ESC scheme is considered to be concave and is assumed to have only one extremum point. Since in the PV system applications, MPPT is employed to extract maximum amount of power from PV panels, therefore, extremum point in this case is maximum point $\left(\theta^{*}\right)$, where at this point $\partial f\left(\theta^{*}\right) / \partial \theta=$ 0 , and also $\partial^{2} f\left(\theta^{*}\right) / \partial \theta^{2}<0$. In ESC algorithm, the output of the nonlinear map is,

$$
y=f(\widehat{\theta}+a \sin (\omega t))
$$

where $a$ and $\omega$ are the amplitude and angular frequency of perturbation signal. Since the perturbation signal assumes to be small, therefore the Taylor expansion of (4) is

$$
y=f(\widehat{\theta})+\frac{d f(\widehat{\theta})}{d \widehat{\theta}} a \sin (\omega t)+\text { H.O.T., }
$$

where H.O.T. stands for higher order terms and $\hat{\theta}$ is the approximation of $\theta^{*}$. The high-pass filtered signal will be,

$$
\begin{aligned}
J \simeq & \mathfrak{Q}^{-1}\left\{\frac{s}{s+\omega_{h}}\right\} * f(\widehat{\theta}) \\
& +\mathfrak{Q}^{-1}\left\{\frac{s}{s+\omega_{h}}\right\} *\left\{\frac{d f(\widehat{\theta})}{d \widehat{\theta}} a \sin (\omega t)\right\} .
\end{aligned}
$$

High-pass filter acts as a derivative opereator in series with a low-pass filter $\left(s\left(1 /\left(s+\omega_{h}\right)\right)\right)$. By multiplying the modulation signal to the resulted signal from the high-pass filter and passing the modulated signal through the low-pass filter and since $\omega_{l}<\omega_{h}$, the output signal of integrator will be

$$
\begin{gathered}
\widehat{\theta} \simeq \frac{a \gamma}{2} \mathbb{Q}^{-1}\left\{\frac{1}{s}\right\} * \mathbb{Q}^{-1}\left\{\frac{\omega_{l}}{s+\omega_{l}}\right\} \\
*\left\{\frac{d}{d t}\left(\frac{d f(\widehat{\theta})}{d \widehat{\theta}}\right)\right\} .
\end{gathered}
$$

Under the assumptions that the amplitude of the sinusoidal perturbation is small and the harmonics of high-pass filter are attenuated by low-pass filter, output of low-pass filter is proportional to the gradient of the nonlinear map with respect to its input and time. Therefore in the neighborhood of the extremum point, the amplitude of the estimated signal is small, since the gradient is small. It can be seen that this amplitude depends on $\gamma$ and $a$.

4.2. Stability Analysis of Averaged ESC Scheme. The averaging method is typically used to analyze the periodic steady state solutions of weakly nonlinear systems. Since the amplitude of perturbation in the ESC scheme is small, this system can be 
evaluated by its averaged model. The averaged form of signal $x(t)$ is,

$$
\overline{x(t)}=\frac{1}{T} \int_{0}^{T} x(t) d t
$$

where $T=2 \pi / \omega$. Therefore the averaged model of ESC scheme is

$$
\begin{gathered}
\overline{\hat{\theta}}=\theta, \\
\bar{y}=\overline{f(\widehat{\theta})}=f(\overline{\hat{\theta}})=f(\theta), \\
\overline{\widehat{\theta}}=\frac{\gamma a}{2} \frac{d}{d t}\left(\frac{d f(\widehat{\theta})}{d \widehat{\theta}}\right) * \mathfrak{L}^{-1}\left\{\mathrm{H}_{\mathrm{LPF}}\right\} * \mathfrak{L}^{-1}\left\{\frac{1}{s}\right\} .
\end{gathered}
$$

On the other hand, in the neighborhood of the extremum point, $y(t)$ can be approximated as,

$$
\begin{gathered}
y \simeq f\left(\theta^{*}\right)+\left.\frac{d f(\theta)}{d \theta}\right|_{\theta=\theta^{*}}\left(\theta-\theta^{*}\right) \\
+\left.\frac{1}{2} \frac{d^{2} f(\theta)}{d \theta}\right|_{\theta=\theta^{*}}\left(\theta-\theta^{*}\right)^{2} .
\end{gathered}
$$

If the difference between the extremum point and averaged point is defined by $\widetilde{\theta}=\bar{\theta}-\theta^{*}$ and since $d f(\theta) /\left.d \theta\right|_{\theta=\theta^{*}}=$ 0 , thus,

$$
\left.\widetilde{y} \simeq \frac{1}{2} \frac{d^{2} f(\theta)}{d \theta^{2}}\right|_{\theta=\theta^{*}} \widetilde{\theta}^{2}
$$

By defining $\left.(1 / 2)\left(d^{2} f(\theta) / d \theta^{2}\right)\right|_{\theta=\theta^{*}}=K$, then, $\left.(d f(\theta) / d \theta)\right|_{\theta=\theta^{*}}=2 K \theta$. Therefore, $\left.(d / d t)(d f(\theta) / d \theta)\right|_{\theta=\theta^{*}}=$ $2(d K / d t) \theta^{*}$. Substituting this relationship in (9) gives,

$$
\overline{\widehat{\theta}}=\left(\gamma a \theta^{*} \frac{d K}{d t}\right) * \mathfrak{Q}^{-1}\left\{\mathrm{H}_{\mathrm{LPF}}\right\} * \mathfrak{Q}^{-1}\left\{\frac{1}{s}\right\} .
$$

Without loss of generality and by assuming that $d K / d t$ is the output signal from a high-pass filter, $\mathrm{H}_{H P F}$, which its cutoff frequency is higher than cutoff frequency of the lowpass filter, $\mathrm{H}_{L P F}$, then (12) can be rewritten as

$$
\overline{\widehat{\theta}}=\left(\gamma a \theta^{*} K\right) \mathfrak{Q}^{-1}\left\{\mathrm{H}_{\mathrm{HPF}}\right\} * \mathfrak{Q}^{-1}\left\{\mathrm{H}_{\mathrm{LPF}}\right\} * \mathfrak{Q}^{-1}\left\{\frac{1}{s}\right\} .
$$

This system can be considered as a feedback system as shown in Figure 7, which $K$ can be considered as a perturbation. The loop gain of this system depends on the demodulation gain, $a$, the integral gain, $\gamma$, and the curvature of nonlinear map, $K$. This result completely matches with the results obtained from other analysis method in the simplified case $[13,31-33]$.

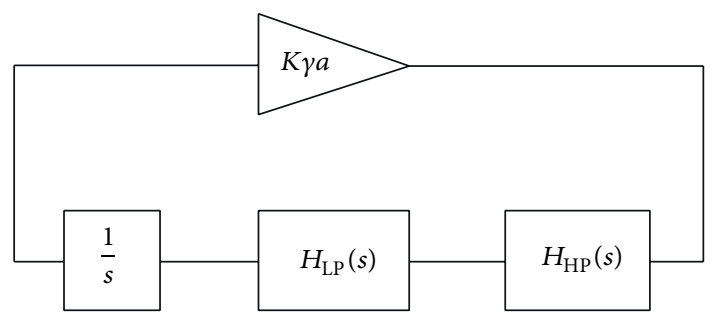

Figure 7: Averaged BICO-ESC scheme.

\section{Analysis of ESC MPPT on PV Panels}

5.1. PV Cell Model. The PV cell $V$ - $I$ curve $v=f(i, G)$ is modeled using the light emitting diode equations [3]:

$$
\begin{gathered}
I=I_{L}-I_{\mathrm{OS}}\left(\exp \left(\frac{q}{A k_{B} T}\right)(V+R I)-1\right), \\
I_{\mathrm{OS}}=I_{\mathrm{OR}}\left(\frac{T}{T_{R}}\right) \exp \left(\frac{q E_{G}}{A k_{B}}\left(\frac{1}{T_{R}}-\frac{1}{R}\right)\right), \\
I_{L}=\frac{G}{1000}\left(I_{\mathrm{SC}}+K_{T, I}\left(T-T_{R}\right)\right), \\
V=\frac{A k_{B} T}{q} \ln \left(\frac{I_{L}-I}{I_{\mathrm{OS}}}+1\right)-R I .
\end{gathered}
$$

Values and definitions for further simulations are shown in Table 2.

5.2. BICO ESC vsersus Regular SISO ESC. In order to compare the qualitative behavior of BICO MPPT with the regular SISO ESC MPPT, the Root-Locus (RL) analysis has been chosen. Clearly, other stability analysis methods are applicable at this point.

Since Root-Locus (RL) method is serving for linear systems, it is important to point out that the curvature constant, $K$, is an uncertain parameter which depends on different factors like the PV panel manufacturer and weather conditions. According to [33], $K \in[-0.5,-5]$.

As seen in Figure 7, the characteristic polynomial of averaged ESC system is $1+a \gamma \mathrm{KH}_{\mathrm{HPF}} \mathrm{H}_{\mathrm{LPF}}$, and to analyze the behavior of this system, Root-Locus (RL) analysis method can be employed. Since there is no command in MATLAB to plot Root-Locus (RL) for BICO transfer function, this equation has been solved for different $a \gamma$ and the roots have been plotted. To compare the behavior of regular SISO ESC with BICO ESC, the roots for this system has been plotted with the same method instead of using "rlocus" command.

Figure 8 shows the comparison between the root locus of averaged ESC using BICO and regular first-order filters. In this root locus plot, the constant gain is assumed to be $a \gamma \theta^{*}=1$ and cutoff frequencies in both filters are assumed to be $\omega_{c}=10 \mathrm{rad} / \mathrm{s}$. Clearly, by using first-order filters in the averaged ESC scheme, for some values of $K$, system has complex poles which cause an oscillatory behavior in the response of the system. On the other side, by using BICO filters, roots of the characteristic polynomial (system poles) are always real numbers for any value of $K$. 
TABLE 2: Values of the considered PV model [3].

\begin{tabular}{lcc}
\hline$T_{R}$ & 298 & Reference temperature \\
$I_{\mathrm{OR}}$ & $2.25 e-6$ & Reverse saturation curent at $T=T_{R}$ \\
$I_{\mathrm{SC}}$ & 3.2 & Short circuit current \\
$E_{G}$ & $1.38 e-19$ & Silicon band gap \\
$A$ & 1.6 & Ideality factor \\
$k_{B}$ & $1.38 e-23$ & Boltzman's constant \\
$q$ & $1.6 e-19$ & Electron charge \\
$R$ & 0.01 & Resistance \\
$K_{T, I}$ & 0.8 & $I_{\mathrm{SC}}$ Temperature coefficient \\
\hline
\end{tabular}

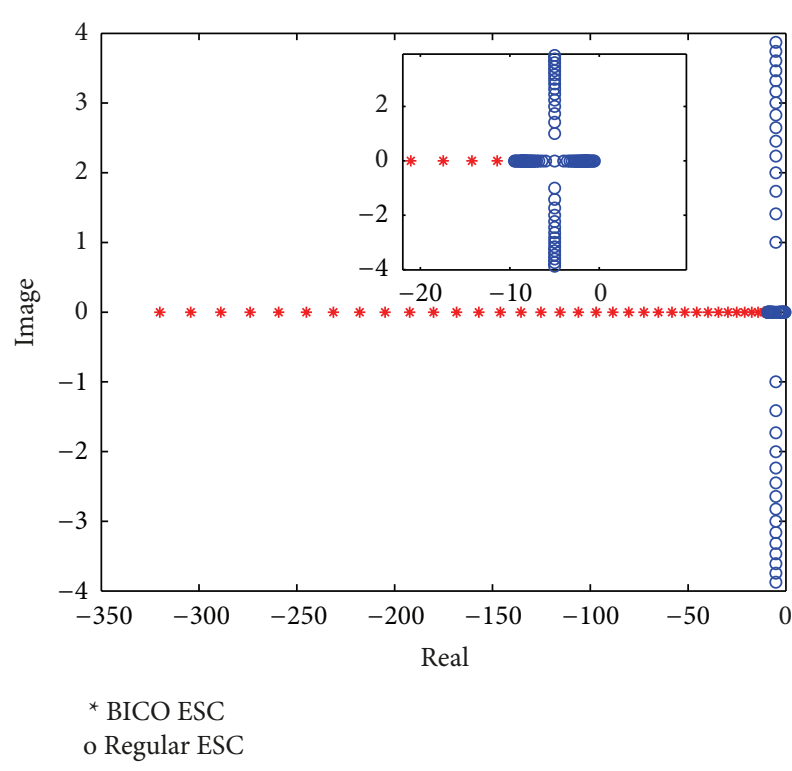

FIGURE 8: Root locus of the averaged BICO and the regular firstorder low-pass filters.

Besides the location of the poles, as can be seen in this figure, for the same value of $K$, BICO filter has farther poles with respect to the origin compared to the first-order filter case. Therefore, the bandwidth of BICO filter is higher than the first-order filter with similar cutoff frequency. Higher bandwidth means faster response for BICO ESC. This feature is justifiable by looking at the frequency response of both filters. Since the bandwidth of BICO is close to the bandwidth of an ideal filter, BICO ESC becomes the fastest achievable MPPT algorithm.

\section{Simulation Illustrations}

Figures 9 and 10 show the maximum power point of a PV panel with the defined parameters in Table 2. As can be seen in these figures, environmental conditions and especially variations in the sun irradiation will change the nonlinear behavior of PV panels. Shadows, cloudy or dusty weather, and temperature variations cause moving of optimal operating point in PV systems. When temperature increases, the maximum output power of PV panels decreases and vice

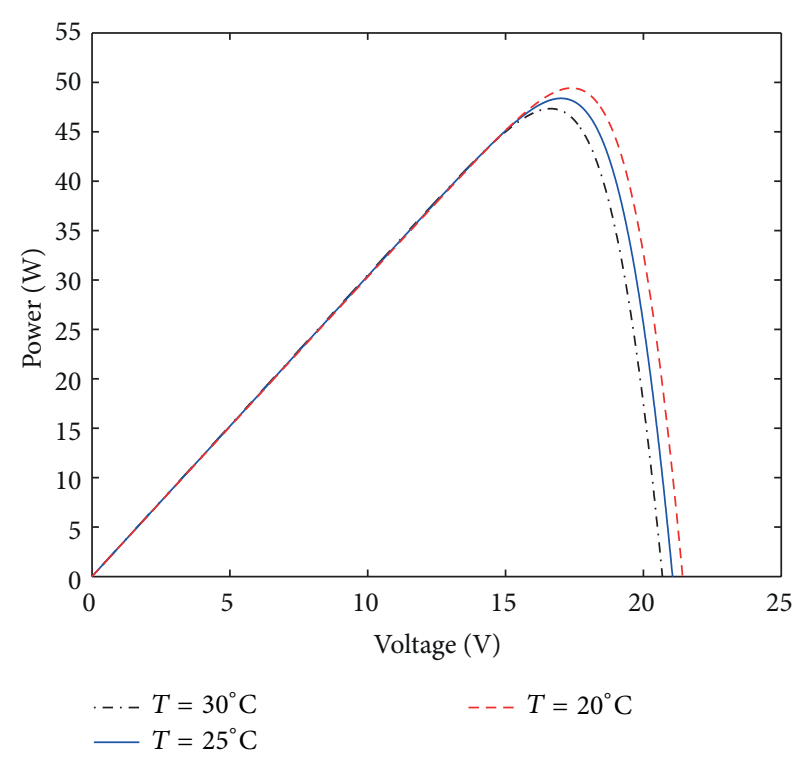

FIgURE 9: $P-V$ chart of considered PV model for different temperatures (irradiation $=1000 \mathrm{~W} / \mathrm{m}^{2}$ ).

versa. In Figure 9 the variations of optimal operating point by changing the environmental temperature from $20^{\circ} \mathrm{C}$ to $30^{\circ} \mathrm{C}$ are illustrated. As Figure 10 presents, variation of peak output power happens in the wider range when sun irradiation changes. Generally, in PV panels, when sun irradiation increases, the output power increases, but when temperature increases, the maximum extractable power reduces.

To compare the proposed MPPT method which is called BICO MPPT with the ESC MPPT, the working conditions of both algorithms have been defined to be same. For all simulations, the ambient temperature is $25^{\circ} \mathrm{C}$ and the irradiation is assumed to be $1000 \mathrm{~W} / \mathrm{m}^{2}$. Also the cutoff frequency of high-pass filters is $\omega_{h}=100 \mathrm{rad} / \mathrm{s}$, and for low-pass filter, this frequency is $\omega_{l}=50 \mathrm{rad} / \mathrm{s}$. Under these conditions, from Figure 9, the maximum amount of power which can be extracted from the simulated PV panel is 48 Watts and this maximum happens around 17 Volts. To implement the BICO MPPT, the discrete approximation of this filter in MathWorks, Inc.s website has been used.

Figure 11 shows the outputs of extremum seeking algorithms resulted from two different MPPT methods. Figure 12 


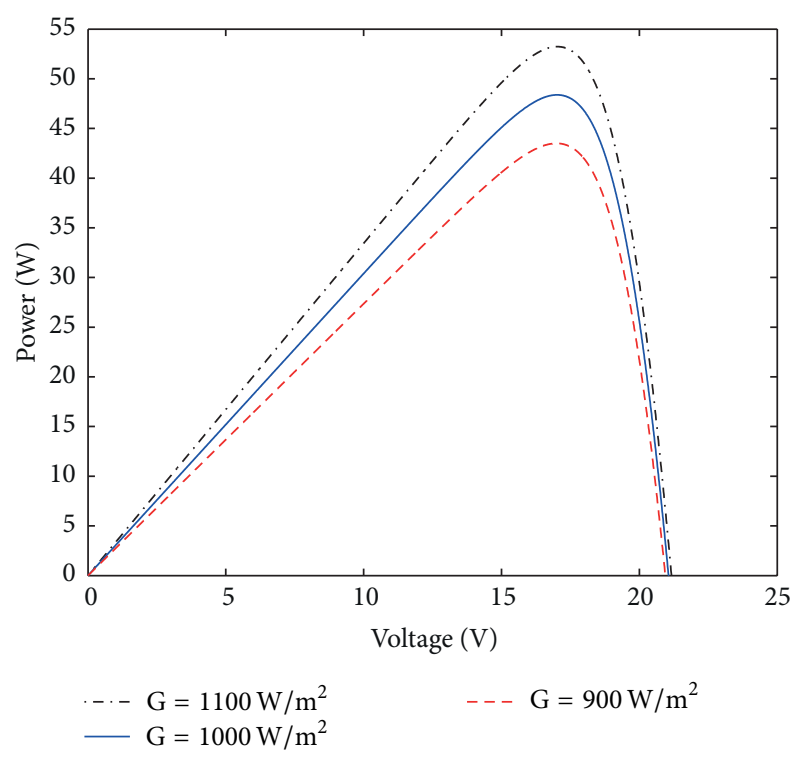

FIgURe 10: $P-V$ chart of considered PV model for different irradiations (temperature $=25^{\circ} \mathrm{C}$ ).

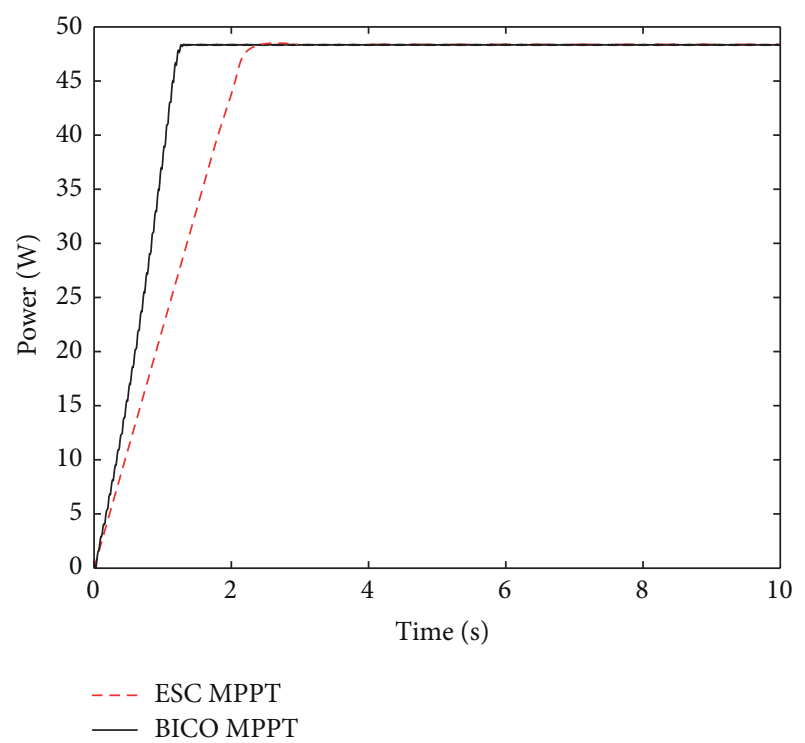

FIGURE 11: Comparison of power tracking in BICO MPPT and ESC MPPT.

represents the maximum voltage tracking in BICO MPPT algorithm and ESC with first-order filters. As expected and proved before, BICO MPPT converges to the peak power point two times faster than the regular ESC MPPT algorithm. Faster convergence speed is due to the higher bandwidth of the averaged BICO system.

Figures 13 and 14 show the performance of the proposed MPPT approach compare to the ESC in the presence of white noise. As can be seen in these figures, in the presences of a white noise (noise power $=0.04$ ), which is considered as the variations in the nonlinear map behavior, $K, \mathrm{BICO}$

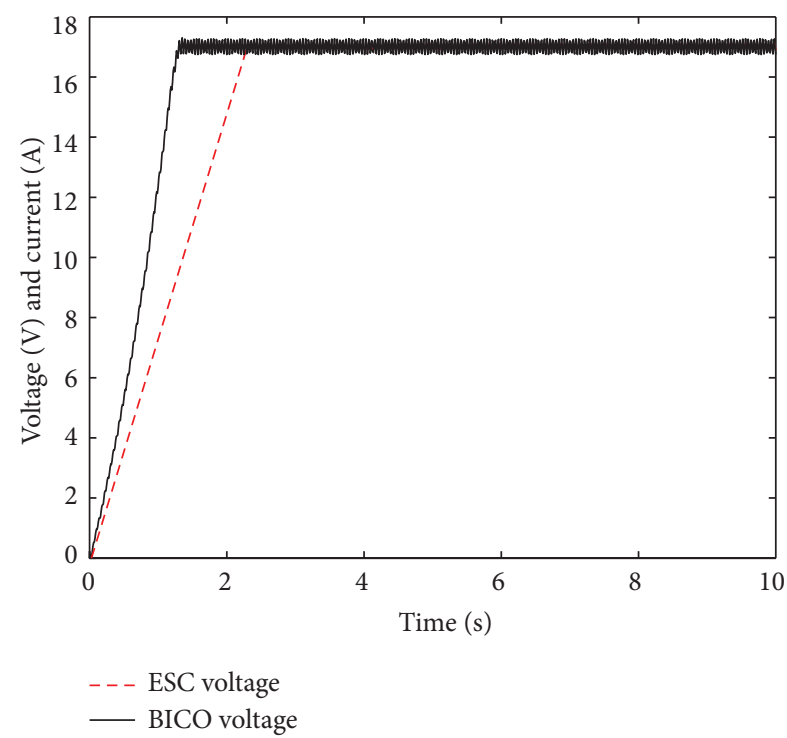

FIGURE 12: Comparison of voltage tracking in BICO MPPT and ESC MPPT.

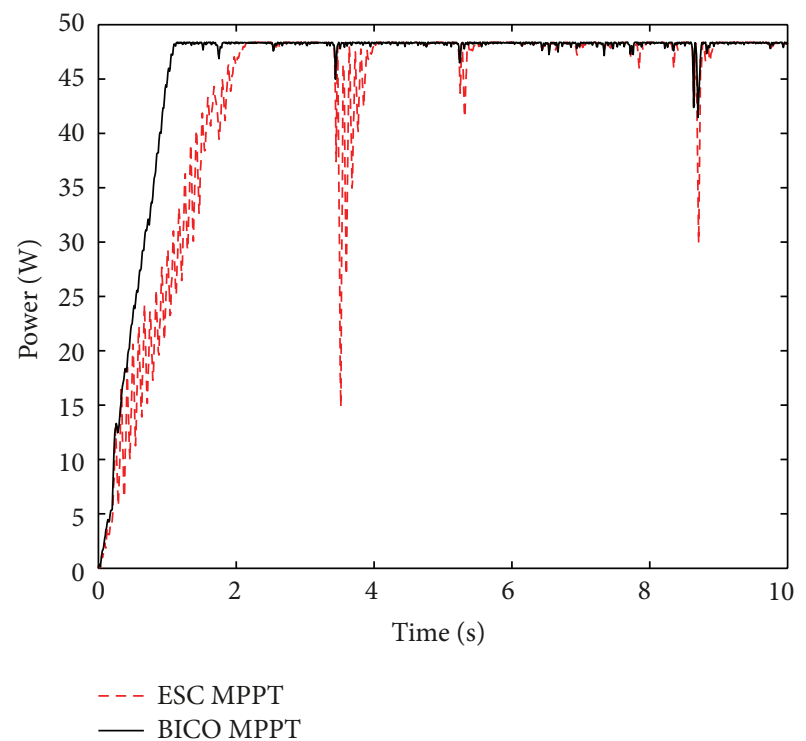

FIgURE 13: Performance of BICO MPPT and ESC MPPT in the presence of a white noise with noise power 0.04 .

MPPT preforms better than ESC MPPT from attenuation and tracking point of view.

Another parameter which is considered in these simulations is the robustness of these two methods to the variations of the system gain. Figures 15(a) and 15(b) illustrate the performance of BICO MPPT and regular ESC MPPT to the gain variations, respectively. From these figures, it can be concluded that, BICO MPPT tolerates higher gain variations, but by increasing the gain of the integrator in ESC MPPT, it starts oscillating. 


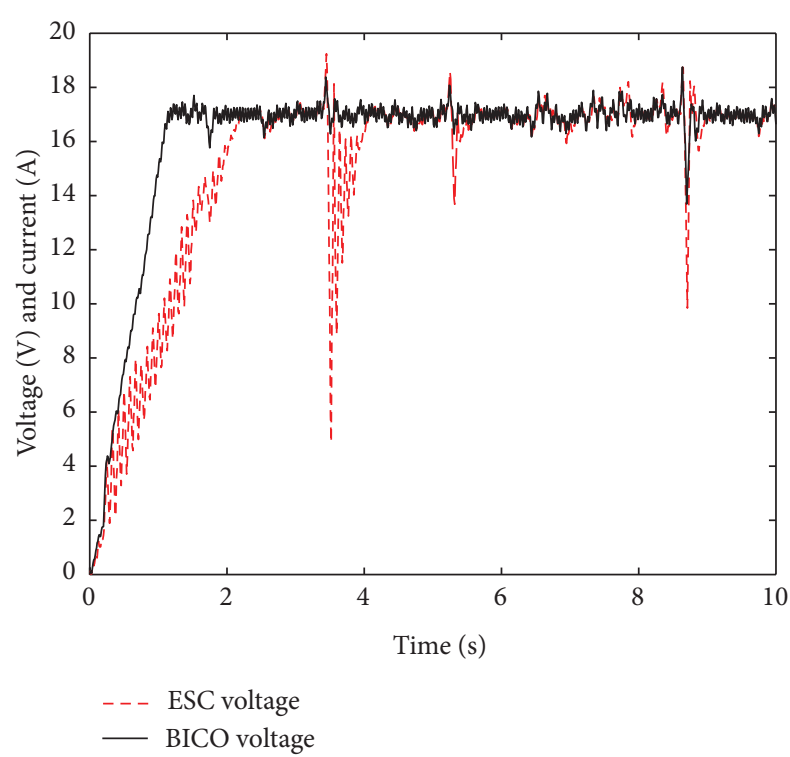

FIGURE 14: Voltage tracking of BICO MPPT and ESC MPPT in the presence of a white noise with noise power 0.04 .

\section{Conclusions}

The ubiquity of using MPPT in the renewable energy systems has stimulated the persistent development of various MPPT algorithms. According to the comparison in this paper, one of the most popular methods of maximum power tracking method is the $\mathrm{P} \& \mathrm{O}$ method. However, this method fails in those situations when there is a need to track the MPPs rapidly.

ESC is a new robust MPPT algorithm which carries all P\&O's benefits and improves its weaknesses. In this paper, a novel ES algorithm has been presented which is called BICO MPPT. This algorithm employs BICO filter in its structure to improve the ESC rise time response even further. BICO filter is a forgotten part of Bode's research that for the first time in this paper was used for PV applications.

BICO filter is the closest filter to the ideal filter and this feature of BICO improves the performance of ESC algorithm. Also, the advantages of BICO has been discussed in this paper. As discussed in this paper, using BICO in the ESC structure increases the bandwidth of the system, which improves the system response.

In addition, applying BICO filter to the ESC algorithm moves all the roots of characteristic polynomial of averaged BICO ESC system to the real axis. Consequently, this system has no pole with imaginary part, and therefore theoretically the system has no oscillatory response by increasing its integrator gain. These poles, however, can have imaginary part by increasing the gain of the system in the regular ESC. This feature increases the robustness of the BICO MPPT compared to regular ESC.

As can be seen in the results, BICO MPPT not only can follow the maximum power point faster than regular ESC, but also it shows more robustness in the presence of disturbance in the system or gain variations.

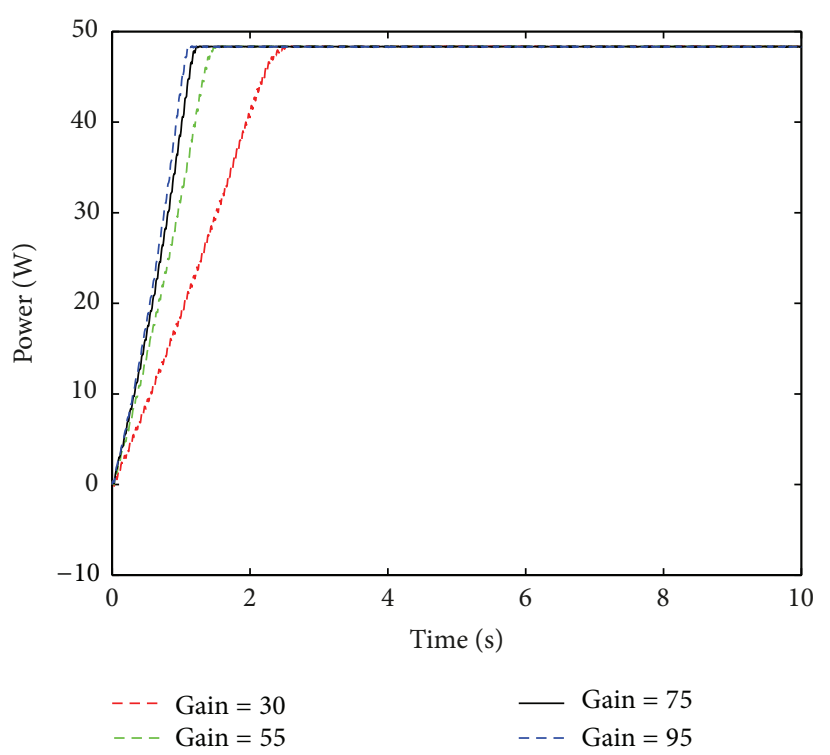

(a)

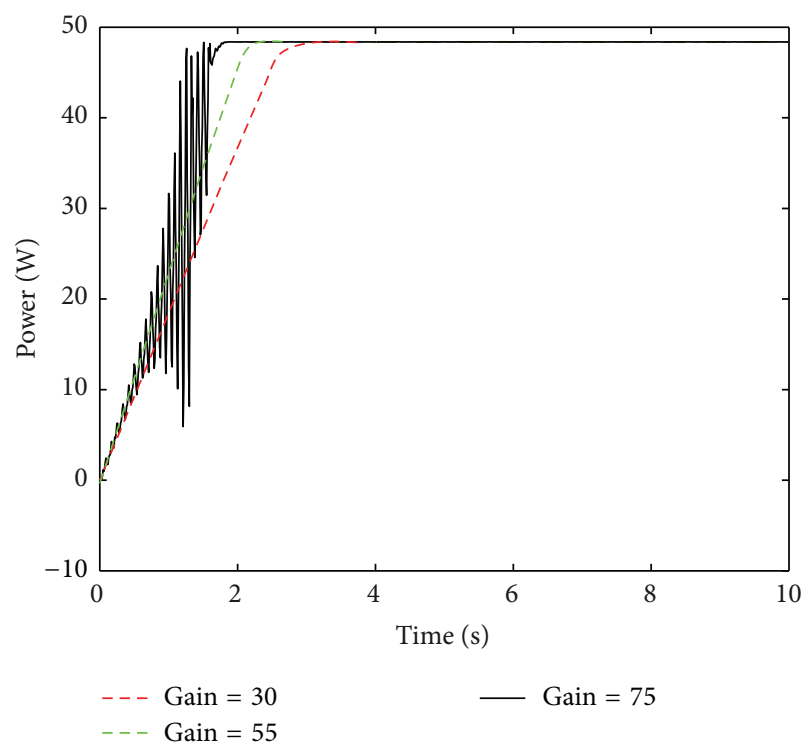

(b)

FIGURE 15: Sensitivity of BICO MPPT and ESC MPPT to the gain variation of integrator.

\section{Conflict of Interests}

The authors declare that there is no conflict of interests regarding the publication of this paper.

\section{References}

[1] B. Bose, P. Szczesny, and R. Steigerwald, "Microcomputer control of a residential photovoltaic power conditioning system," IEEE Transactions on Industry Applications, vol. 21, no. 5, pp. 1182-11191, 1985.

[2] R. A. Mastromauro, M. Liserre, T. Kerekes, and A. Dell'Aquila, "A single-phase voltage-controlled grid-connected photovoltaic system with power quality conditioner functionality," IEEE 
Transactions on Industrial Electronics, vol. 56, no. 11, pp. 44364444, 2009.

[3] S. L. Brunton, C. W. Rowley, S. R. Kulkarni, and C. Clarkson, "Maximum power point tracking for photovoltaic optimization using ripple-based extremum seeking control," IEEE Transactions on Power Electronics, vol. 25, no. 10, pp. 2531-2540, 2010.

[4] T. Esram and P. L. Chapman, "Comparison of photovoltaic array maximum power point tracking techniques," IEEE Transactions on Energy Conversion, vol. 22, no. 2, pp. 439-449, 2007.

[5] J. J. Nedumgatt, K. B. Jayakrishnan, S. Umashankar, D. Vijayakumar, and D. P. Kothari, "Perturb and observe MPPT algorithm for solar PV systems-modeling and simulation," in Proceedings of the Annual IEEE India Conference: Engineering Sustainable Solutions (INDICON '11), pp. 1-6, December 2011.

[6] R. Alonso, P. Ibáñez, V. Martínez, E. Román, and A. Sanz, “An innovative perturb, observe and check algorithm for partially shaded PV systems," in Proceedings of the 13th European Conference on Power Electronics and Applications (EPE '09), pp. 1-8, September 2009.

[7] N. Femia, G. Petrone, G. Spagnuolo, and M. Vitelli, “Optimization of perturb and observe maximum power point tracking method," IEEE Transactions on Power Electronics, vol. 20, no. 4, pp. 963-973, 2005.

[8] G. J. Kish, J. J. Lee, and P. W. Lehn, "Modelling and control of photovoltaic panels utilising the incremental conductance method for maximum power point tracking," IET Renewable Power Generation, vol. 6, no. 4, pp. 259-266.

[9] A. Safari and S. Mekhilef, "Simulation and hardware implementation of incremental conductance MPPT with direct control method using cuk converter," IEEE Transactions on Industrial Electronics, vol. 58, no. 4, pp. 1154-1161, 2011.

[10] H. Malek, S. Dadras, Y. Q. Chen, R. Burt, and J. Cook, "Maximum power point tracking techniques for efficient photovoltaic microsatellite power supply system," in Proceeding of the Small Satellite Conference, Logan, Utah, USA, 2012.

[11] H. Malek, S. Dadras, and Y. Q. Chen, "A fractional order maximum power point tracker: Stability analysis and experiments," in Proceeding of the IEEE 51st Annual Conference on Decision and Control (CDC '12), pp. 6861-6866, Maui, Hawaii, USA, 2012.

[12] R. Faranda and S. Leva, "Energy comparison of MPPT techniques for PV systems," WSEAS Transactions on Power Systems, vol. 3, no. 6, pp. 1-6, 2008.

[13] M. Krstić and H.-H. Wang, "Stability of extremum seeking feedback for general nonlinear dynamic systems," Automatica, vol. 36, no. 4, pp. 595-601, 2000.

[14] M. Guay, D. Dochain, and M. Perrier, "Adaptive extremum seeking control of continuous stirred tank bioreactors with unknown growth kinetics," Automatica, vol. 40, no. 5, pp. 881888, 2004.

[15] G. Bastin, D. Nešić, Y. Tan, and I. Mareels, "On extremum seeking in bioprocesses with multivalued cost functions," Biotechnology Progress, vol. 25, no. 3, pp. 683-689, 2009.

[16] S. Drakunov, U. Ozguner, P. Dix, and B. Ashrafi, "ABS control using optimum search via sliding modes," IEEE Transactions on Control Systems Technology, vol. 3, no. 1, pp. 79-85, 1995.

[17] D. Popović, M. Janković, S. Magner, and A. Teel, "Extremum seeking methods for optimization of variable cam timing engine operation," in Proceeding of the American Control Conference, pp. 3136-3141, Denver, Colo, USA, June 2003.

[18] K. S. Peterson and A. G. Stefanopoulou, "Extremum seeking control for soft landing of an electromechanical valve actuator," Automatica, vol. 40, no. 6, pp. 1063-1069, 2004.
[19] H.-H. Wang, S. Yeung, and M. Krstić, "Experimental application of extremum seeking on an axial-flow compressor," IEEE Transactions on Control Systems Technology, vol. 8, no. 2, pp. 300-309, 2000.

[20] C. G. Mayhew, R. G. Sanfelice, and A. R. Teel, "Robust source-seeking hybrid controllers for autonomous vehicles," in Proceedings of the American Control Conference (ACC '07), pp. 1185-1190, New York, NY, USA, July 2007.

[21] E. Biyik and M. Arcak, "Gradient climbing in formation via extremum seeking and passivity-based coordination rules," in Proceedings of the 46th IEEE Conference on Decision and Control (CDC '07), pp. 3133-3138, New Orleans, La, USA, December 2007.

[22] P. Ögren, E. Fiorelli, and N. E. Leonard, "Cooperative control of mobile sensor networks: adaptive gradient climbing in a distributed environment," IEEE Transactions on Automatic Control, vol. 49, no. 8, pp. 1292-1302, 2004.

[23] P. M. Dower, P. M. Farrell, and D. Nešić, "Extremum seeking control of cascaded Raman optical amplifiers," IEEE Transactions on Control Systems Technology, vol. 16, no. 3, pp. 396-407, 2008.

[24] J. Sternby, "Extremum control systems: an area for adaptive control," in Proceedings of the International Symposium on Adaptive Systems, vol. 24, pp. 151-160, San Francisco, Calif, USA, 1980.

[25] K. B. Ariyur and M. Krstic, Real-Time Optimization by Extremum-Seeking Control, Wiley-Interscience, 2003.

[26] K. J. Astrom and B. Wittenmark, Adaptive Control, AddisonWesley, 2nd edition, 1995.

[27] Y. Luo, T. Zhang, B. Lee, C. Kang, and Y. Q. Chen, “Disturbance observer design with bode's ideal cut-off filter in hard-disc-drive servo system," Mechatronics, vol. 23, no. 7, pp. 856-862, 2013.

[28] L. E. Olivier, I. K. Craig, and Y. Q. Chen, "Fractional order and BICO disturbance observers for a run-of-mine ore milling circuit," Journal of Process Control, vol. 22, no. 1, pp. 3-10, 2012.

[29] T. T. Hartley and C. F. Lorenzo, "Optimal fractional-order damping," in Proceedings of the ASME Design Engineering Technical Conferences and Computers and Information in Engineering Conference, pp. 737-743, Chicago, Ill, USA, September 2003.

[30] Oberhettinger and Baddi, Tables of Laplace Transforms, Springer, Berlin, Germany, 1973.

[31] Y. Tan, D. Nešić, and I. Mareels, "On stability properties of a simple extremum seeking scheme," in Proceedings of the 45th IEEE Conference on Decision and Control (CDC '06), pp. 28072812, December 2006.

[32] Y. Tan, D. Nešić, and I. Mareels, "On non-local stability properties of extremum seeking control," Automatica, vol. 42, no. 6, pp. 889-903, 2006.

[33] R. Leyva, C. Olalla, H. Zazo et al., "Mppt based on sinusoidal extremum-seeking control in pv generation," International Journal of Photoenergy, vol. 98, no. 4, pp. 529-542, 2011.

[34] R. Leyva, P. Artillan, C. Cabal, B. Estibals, and C. Alonso, "Dynamic performance of maximum power point tracking circuits using sinusoidal extremum seeking control for photovoltaic generation," International Journal of Electronics, vol. 98, no. 4, pp. 529-542, 2011. 

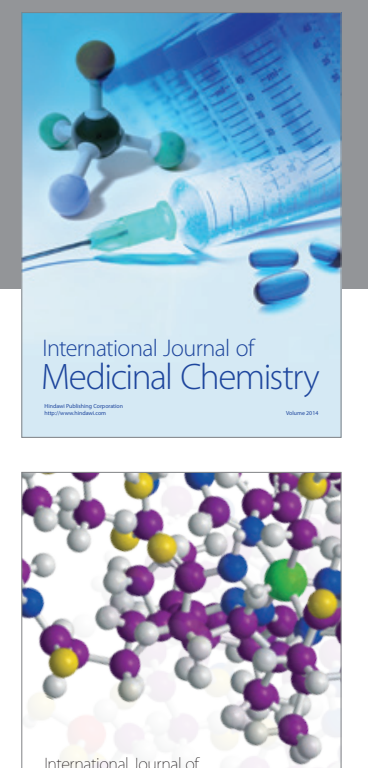

\section{Carbohydrate} Chemistry

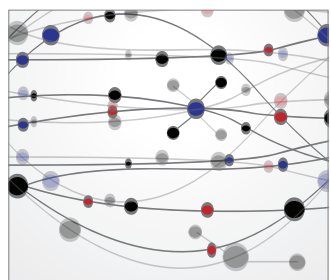

The Scientific World Journal
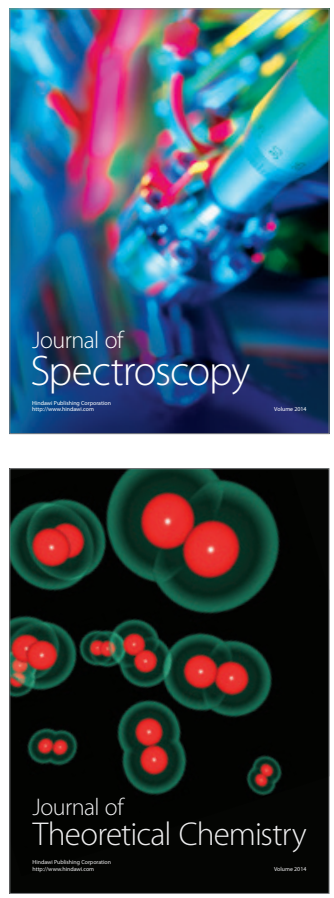
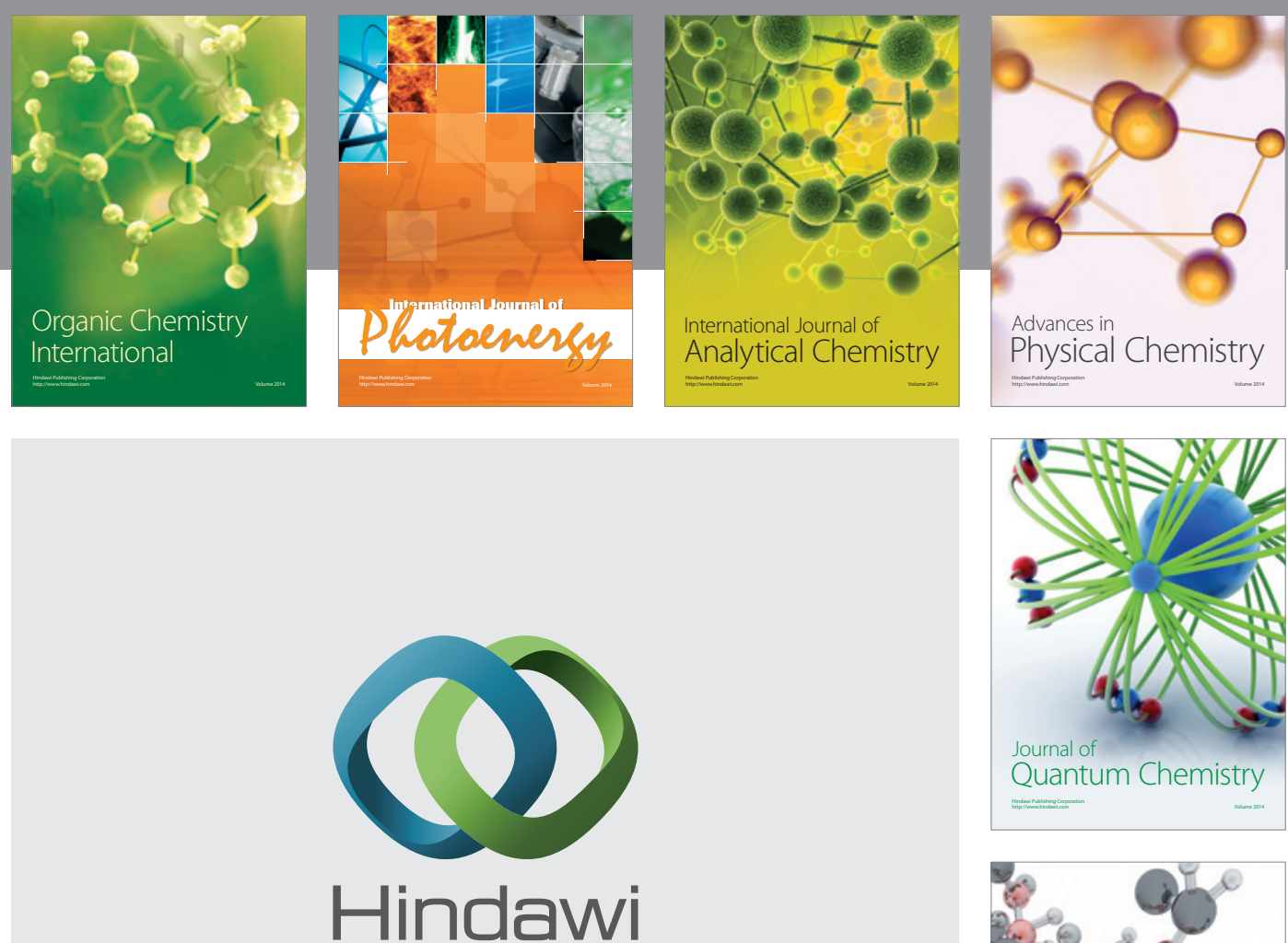

Submit your manuscripts at

http://www.hindawi.com

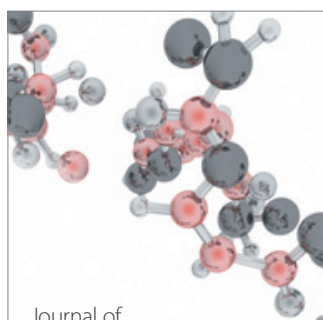

Analytical Methods

in Chemistry

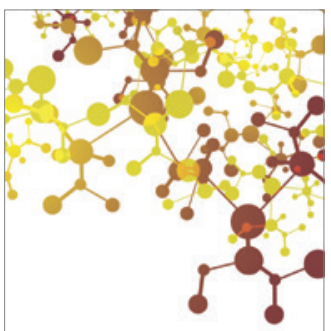

Journal of

Applied Chemistry

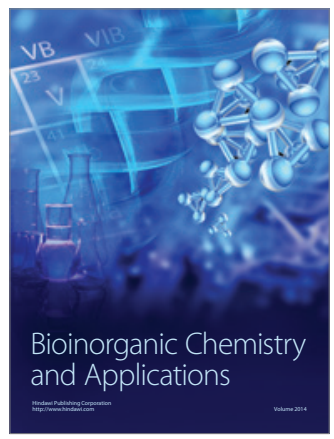

Inorganic Chemistry
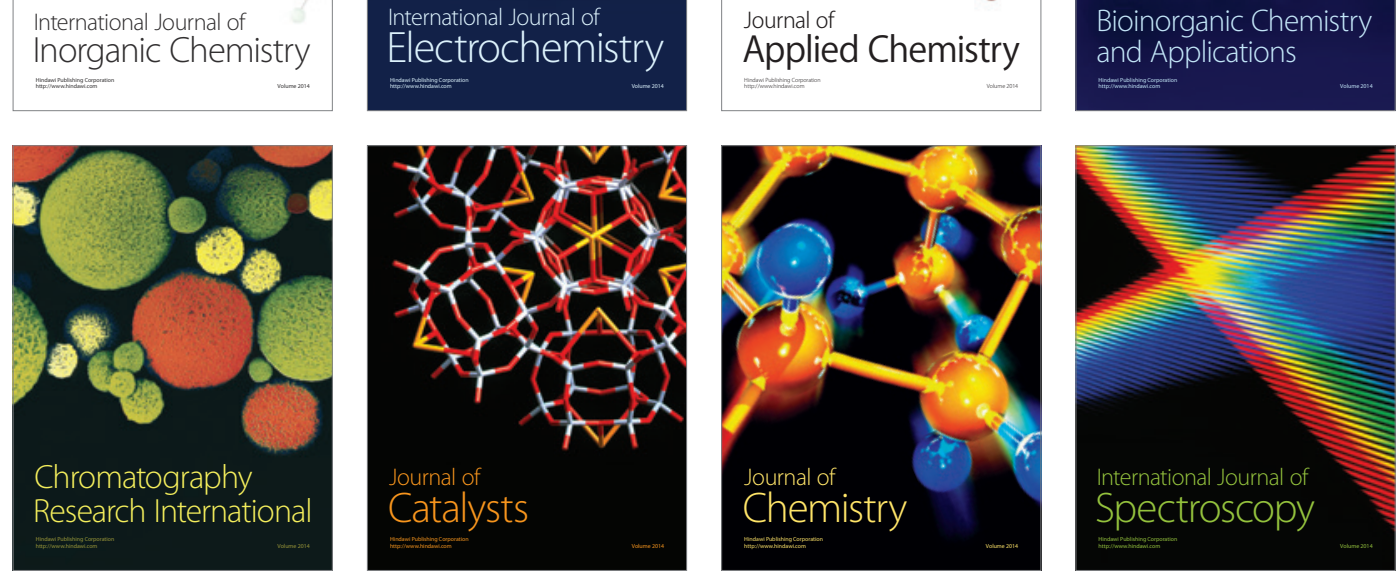